\title{
Plasma versus Drude Modeling of the Casimir Force: Beyond the Proximity Force Approximation
}

\author{
Michael Hartmann, ${ }^{1}$ Gert-Ludwig Ingold, ${ }^{1}$ and Paulo A. Maia Neto ${ }^{2}$ \\ ${ }^{1}$ Universität Augsburg, Institut für Physik, 86135 Augsburg, Germany \\ ${ }^{2}$ Instituto de Física, Universidade Federal do Rio de Janeiro, CP 68528, Rio de Janeiro, Rio de Janeiro 21941-909, Brazil
}

(Received 11 May 2017; published 24 July 2017)

\begin{abstract}
We calculate the Casimir force and its gradient between a spherical and a planar gold surface. Significant numerical improvements allow us to extend the range of accessible parameters into the experimental regime. We compare our numerically exact results with those obtained within the proximity force approximation (PFA) employed in the analysis of all Casimir force experiments reported in the literature so far. Special attention is paid to the difference between the Drude model and the dissipationless plasma model at zero frequency. It is found that the correction to PFA is too small to explain the discrepancy between the experimental data and the PFA result based on the Drude model. However, it turns out that for the plasma model, the corrections to PFA lie well outside the experimental bound obtained by probing the variation of the force gradient with the sphere radius [D. E. Krause et al., Phys. Rev. Lett. 98, 050403 (2007)]. The corresponding corrections based on the Drude model are significantly smaller but still in violation of the experimental bound for small distances between plane and sphere.
\end{abstract}

DOI: 10.1103/PhysRevLett.119.043901

The last decades have witnessed a surge in precise measurements of the Casimir interaction [1-4]. Instead of the theoretical paradigm of two parallel metallic plates [5], most experiments adopt the plane-sphere geometry to avoid misalignment. This geometry is in principle amenable to an exact description by the scattering approach [6-8]. The Casimir interaction energy was calculated in the plane-sphere geometry at zero temperature for perfect $[9,10]$ and real metals [11], as well as for finite temperatures [12,13] and in the high-temperature limit [14-16]. In spite of those recent theoretical developments, the analysis of the plane-sphere experiments to date has relied exclusively on the heuristic proximity force approximation (PFA), also known as Derjaguin approximation [17].

Experimentally a measurable force signal requires the radius $R$ of the sphere to be much larger than the separation $L$ between sphere and plane; cf. Fig. 1(a). For probing distances in the micrometer range, coated macroscopic lenses with radii of more than $10 \mathrm{~cm}$ leading to aspect ratios $R / L \sim 10^{5}$ were used $[18,19]$. In experiments exploring the Casimir interaction in the submicrometer regime smaller aspect ratios of $R / L \sim 10^{3}$ were realized [20-25]. However, up to now these aspect ratios were out of reach for numerically exact computations. Within the scattering approach, the required number of multipoles scales like $R / L$. In practice, the number of multipoles so far was limited to $\ell \sim 500$ allowing for aspect ratios of $R / L \sim 100$ [26]. Such calculations are capable of addressing recently proposed experiments based on optical tweezers as a tool for probing femtonewton Casimir forces well outside the validity of PFA [27], but are not suited for describing typical Casimir force experiments.
In this Letter, we significantly extend the range of numerically accessible aspect ratios to values of $R / L \sim$ $10^{3}$ and report on results for the Casimir force and force gradient. We take the parameters corresponding to the experiments in Refs. [20-23] with gold surfaces at room temperature, but our approach also opens the way to calculate exact results for a variety of recent experiments with similar aspect ratios and different materials like magnetic materials [24] and layered surfaces [25]. The key ingredients allowing us to treat experimentally relevant aspect ratios are a new symmetrical representation of the round-trip scattering operator and a state-of-the-art algorithm for evaluating determinants of hierarchical matrices.

Although PFA is expected to provide the correct leading divergence in the limit $R / L \rightarrow \infty$, the magnitude of the correction to PFA under real experimental conditions was not known until now. In Ref. [20], the force gradient
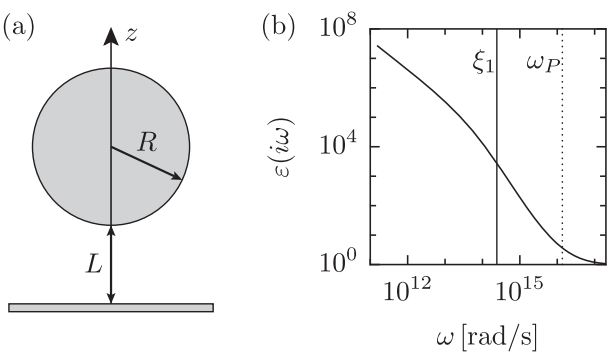

FIG. 1. (a) Sphere of radius $R$ separated from the plane by a distance $L$. (b) Frequency dependence of the permittivity of gold used in the numerical calculations. The vertical solid line indicates the first Matsubara frequency $\xi_{1}$ while the dotted line indicates the plasma frequency $\omega_{P}$. 
variation with $R$ was probed experimentally, and an upper bound for the correction was derived. On the theoretical side, recent advances were based either on asymptotic expansions valid in the particular case of perfect reflectors at zero temperature [28] or on the derivative expansion approach [29-31]. The latter relies on a resummation of the perturbative expansion around the planar geometry. Its application to compact objects like the sphere thus relies on the assumption that only the lower hemisphere contributes when $R / L \gg 1$ [32]. Moreover, the derivative expansion requires analyticity of the perturbative kernel, a condition not met for the zero-frequency contribution when taking the plasma model [33].

None of these approaches allow for a direct comparison with the experimental bound derived in Ref. [20], since they provide only the leading-order correction to the PFA result. In fact, the next-to-leading-order correction might be comparable to the leading-order one for typical experimental aspect ratios $R / L \sim 10^{3}$, as for instance in the case of Drude metals at high temperatures [15]. A recent proposal combines the leading-order correction for the positive Matsubara frequencies and the exact result for the zero-frequency contribution in the case of Drude metals [34]. However, no such result is available for the plasma model. Here, we present exact numerical results for the force and the force gradient taking the parameters of [20] and either the Drude or plasma prescriptions for the Matsubara zero-frequency contribution. Both prescriptions violate the experimental bound for the correction to the force gradient at sphere-plane distances below $400 \mathrm{~nm}$, but the corrections for the Drude prescription are significantly smaller.

In the scattering approach the Casimir interaction free energy is given by $[6,7]$

$$
\mathcal{F}=\frac{k_{B} T}{2} \sum_{n=-\infty}^{\infty} \log \operatorname{det}\left[1-\mathcal{M}\left(\left|\xi_{n}\right|\right)\right]
$$

where $\mathcal{M}(\xi)$ denotes the round-trip operator at imaginary frequency $\xi$ and the Matsubara frequencies $\xi_{n}=$ $2 \pi n k_{B} T / \hbar$ are proportional to the temperature $T$. For reasons explained below and in contrast to the common choice, we adopt a symmetrized form of the round-trip operator

$$
\mathcal{M}(\xi)=\sqrt{\mathcal{R}_{S}} e^{-\mathcal{K}(L+R)} \mathcal{R}_{P} e^{-\mathcal{K}(L+R)} \sqrt{\mathcal{R}_{S}} .
$$

The reflection operator at the plane $\mathcal{R}_{P}$ is diagonal in the plane-wave basis. Its matrix elements are given by the Fresnel coefficients $r_{p}(k, i \xi)$, where the polarization $p$ can either be transverse magnetic (TM) or transverse electric (TE), and $k$ denotes the projection of the wave vector onto the plane. The translation operator $\exp [-\mathcal{K}(L+R)]$ covers the distance between the plane and the center of the sphere along the $z$ direction; cf. Fig. 1(a). $\mathcal{K}$ is diagonal in the plane-wave basis as well, with matrix elements $\sqrt{k^{2}+\xi^{2} / c^{2}}$. Finally, the reflection operator $\mathcal{R}_{S}$ is diagonal in the multipole basis with matrix elements given by the Mie coefficients $a_{\ell}(i \xi), b_{\ell}(i \xi)$ [35].

Our particular choice (2) for the round-trip operator $\mathcal{M}$ is a key ingredient to push the numerics into the experimentally accessible parameter range. First, it avoids illconditioned matrices with elements differing by more than one hundred orders of magnitude that render a fast and stable evaluation of the free energy (1) difficult [26]. In fact, numerical tests suggest that with the round-trip operator of the form (2), $1-\mathcal{M}$ becomes diagonally dominant. Second, it turns out that the matrix $\mathcal{M}$ can be hierarchically factored. This means that although the matrix is not sparse, it can be efficiently approximated by considering only a subset of all matrix elements. The error caused by this approximation can be made negligibly small. We efficiently compute the determinants using the implementation [36] of an algorithm designed for hierarchical off-diagonal lowrank matrices [37]. Another key ingredient is a fast computation of Legendre polynomials $P_{\ell}(z)$ [38] to efficiently evaluate associated Legendre functions $P_{\ell}^{m}(z)$ arising in the change between multipole and plane-wave basis. These numerical improvements allow us to calculate the plane-sphere Casimir energy up to aspect ratios $R / L \sim$ $4 \times 10^{3}$ requiring multipole orders $\ell \sim 2 \times 10^{4}$.

While the details of our numerical approach will be discussed elsewhere [39], it is worth pointing out checks supporting the validity of our results. We have found agreement with the exact analytical result for the Drude model in the high-temperature limit [15] and the leading correction to PFA for perfect reflectors at $T=0[28,30]$. Finally, for the Drude prescription our results shown below in Fig. 2(b) are consistent with those obtained from the derivative expansion [31].

Here, we will focus on gold surfaces [20-23] at room temperature $T=295 \mathrm{~K}$. The permittivity of gold at imaginary frequencies entering the reflection coefficients can be derived from tabulated optical data [40] as explained in Ref. [41]. As shown in Fig. 1(b), the frequency range covered by this procedure includes all required Matsubara frequencies except for $n=0$.

For the treatment of the zero-frequency contribution, two models have been used in the analysis of experiments, the Drude model and the plasma model. Since for $n=0$ no polarization mixing occurs [42], TM and TE modes contribute independently. The TM mode for both models is perfectly reflected by plane and sphere, and thus its contribution to the Casimir free energy only depends on $R / L$. In contrast, the contribution for the TE mode depends on the model chosen. While for the Drude model no contribution arises [43], the contribution for the plasma model is nonvanishing and also depends on the plasma frequency $\omega_{P}[14]$. 


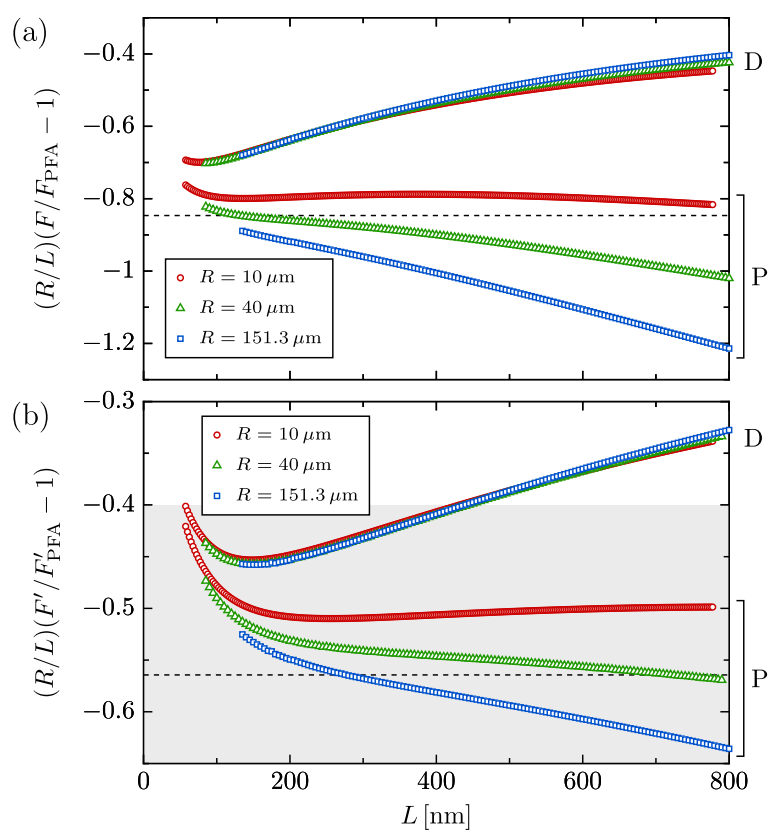

FIG. 2. Beyond-PFA corrections to (a) the force and (b) the force gradient are shown as function of the distance between sphere and plane. We multiply the correction by the aspect ratio $R / L$. The upper three and lower three lines refer to the Drude $(D)$ and plasma $(P)$ prescription, respectively. The dashed horizontal lines indicate the results for perfect reflectors at zero temperature, corresponding to the constant coefficients $\beta_{T=0}^{\mathrm{PR}}$ and $\beta_{T=0}^{\prime \mathrm{PR}}$ for the force and force gradient, respectively, as defined in the text. In the lower panel, the grey area marks the parameter range for the force gradient excluded experimentally at the $95 \%$ confidence level [20].

It has been argued that even in the plasma model the TE mode does not contribute to the Casimir free energy [44]. Nevertheless, for a number of experiments agreement of the results with the plasma prescription just introduced was found [21-25]. In a few cases experimental support for the Drude prescription was claimed $[19,45]$, but also questioned [46]. All in all, there is no agreement yet on how the zero-frequency contribution should be accounted for.

For gold, we find from the optical data the plasma frequency $\omega_{P}=9 \mathrm{eV}$ which differs slightly from the value $\omega_{P}=8.9 \mathrm{eV}$ used to analyze the experiment in Ref. [21]. For the zero-frequency contribution in the Drude case, we use the analytical result derived with the help of bispherical coordinates [15] instead of performing a numerical evaluation.

We calculate the Casimir force $F=-\partial \mathcal{F} / \partial L$ and compare the results with the proximity force approximation $F_{\mathrm{PFA}}=2 \pi R \mathcal{F}_{\mathrm{PP}}(L) / A$, where $\mathcal{F}_{\mathrm{PP}}(L) / A$ is the Casimir free energy per unit area for parallel plates at a distance $L$. Within the derivative expansion approach, the leading correction to PFA is of the form

$$
\frac{F}{F_{\mathrm{PFA}}}-1=\beta(L) \frac{L}{R}+\cdots,
$$

with the coefficient $\beta(L)$ independent of $R$ [29]. The subleading corrections might contain logarithmic terms, as for instance in the case of high temperatures [15]. For the special case of perfect reflectors and zero temperature, the coefficient $\beta$ is independent of $L$ and given by $\beta_{T=0}^{\mathrm{PR}}=$ $1 / 6-10 / \pi^{2} \approx-0.847[28,30]$.

In order to test (3) and obtain a numerical approximation for $\beta(L)$, we plot in Fig. 2(a) the variation of $(R / L)\left(F / F_{\mathrm{PFA}}-1\right)$ with the distance $L$ for radii $R=10,40$, and $151.3 \mu \mathrm{m}$. The upper three curves correspond to the Drude prescription $(D)$ while the lower three curves correspond to the plasma prescription $(P)$. The dashed line indicates the value of $\beta_{T=0}^{\mathrm{PR}}$. According to (3), the correction to the force scaled with $R / L$ should approach $\beta(L)$ and be independent of $R$ for sufficiently small values of $L / R$. This is indeed the case when considering the Drude prescription for $L \lesssim 400 \mathrm{~nm}$ and the sphere radii shown in Fig. 2. As the distance increases, the curves representing different radii start to deviate from each other. This behavior can be associated with the contribution of subleading corrections, which become comparatively more important as $L$ increases. At a fixed temperature, larger distances result in an increase of the relative contribution of the zero Matsubara frequency [15], for which the subleading correction is comparable to the leading one for the parameters represented in the figure.

On the other hand, when taking the plasma prescription for the zero-frequency contribution, the curves corresponding to different values of $R$, shown in the lower part of Fig. 2(a), are well separated from each other, indicating that the correction to PFA is not of the form (3) in this case. The contributions from the Matsubara frequencies with $n \neq 0$ are exactly the same for the two models. Hence the difference shown in Fig. 2(a) is entirely due to the TE zero-frequency contribution present in the plasma prescription but not in the Drude prescription.

The zero-frequency contribution becomes relatively more important as $L$ increases, separating the plasma curves from each other and from the Drude curve. The derivative expansion approach fails in the plasma model at finite temperatures precisely because of the nonanalytical nature of the perturbative kernel corresponding to the TE zero-frequency contribution [33], thus resulting in the structure shown in the lower part of Fig. 2(a). We also remark that in contrast to what is frequently believed, the case of perfect reflectors at zero temperature, indicated by the horizontal dashed line in Fig. 2(a), does not provide an upper bound for the magnitude of the force correction for $L \gtrsim 100 \mathrm{~nm}$ due to the contribution of the TE zerofrequency mode in the plasma model.

The magnitude of the correction to PFA was experimentally investigated in Ref. [20]. The Casimir force gradient $F^{\prime}=-\partial^{2} \mathcal{F} / \partial L^{2}$ was measured for different sphere radii, and a linear dependence with $1 / R$ similar to (3) was proposed 

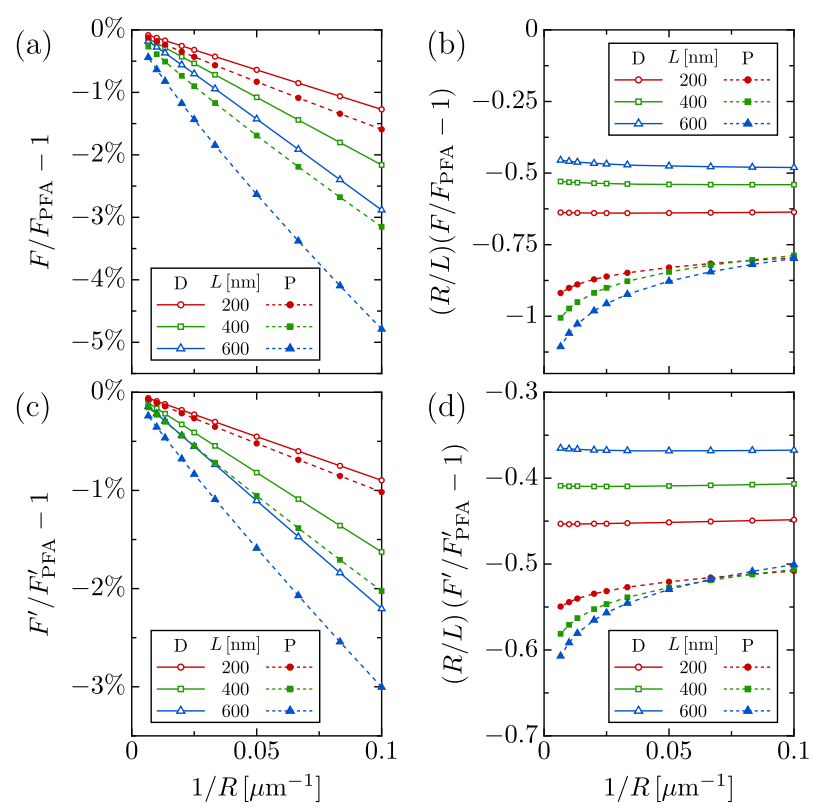

FIG. 3. Beyond-PFA corrections to the (a),(b) force and (c),(d) force gradient are shown as function of the inverse sphere radius. While in (a) and (c) the relative corrections are displayed, the data in (b) and (d) have been scaled by $R / L$. The sphere-plane distances are $L=200 \mathrm{~nm}(\circ), 400 \mathrm{~nm}(\square)$, and $600 \mathrm{~nm}(\triangle)$. Solid lines with open symbols refer to the Drude prescription while dashed lines with filled symbols refer to the plasma prescription.

$$
\frac{F^{\prime}}{F_{\mathrm{PFA}}^{\prime}}-1=\beta^{\prime}(L) \frac{L}{R}+\cdots
$$

While the authors of [20] were unable to measure the correction term, they nonetheless derived the upper bound $\left|\beta^{\prime}(L)\right|<0.4$ at the $95 \%$ confidence level for $L$ in the interval between 150 and $300 \mathrm{~nm}$. In Fig. 2(b), we plot the variation of $(R / L)\left(F^{\prime} / F_{\mathrm{PFA}}^{\prime}-1\right)$ with the distance $L$ for the same values of $R$ used in Fig. 2(a). This quantity provides an approximation for the coefficient $\beta^{\prime}(L)$ as long as the results are independent of $R$. The shaded area represents values for the correction excluded by the experiment [20] while the dashed line indicates the correction for perfect reflectors at $T=0$ given by $\beta_{T=0}^{\prime \mathrm{PR}}=(2 / 3) \beta_{T=0}^{\mathrm{PR}} \approx$ $-0.564[28,30]$.

For $L \lesssim 400 \mathrm{~nm}$ the Drude as well as the plasma prescription violate the experimental bound, although the maximum violation for the Drude prescription at $L \approx$ $150 \mathrm{~nm}$ corresponding to $\beta^{\prime} \approx-0.46$ is significantly smaller than the violation found for the plasma prescription. Note, however, that the plasma and Drude curves get closer to each other as the distance decreases below $200 \mathrm{~nm}$, as expected in the low temperature regime, with the zero frequency providing a relatively smaller contribution.

As in the discussion of the correction to the force, the results for different radii shown in Fig. 2(b) are very close to each other and to the results obtained within the derivative expansion approach [31,34] when taking the Drude prescription. In this case, our results show that subleading corrections are negligible for the experimental conditions of Refs. [20-23], which correspond to aspect ratios in the range $R / L \sim 10^{2}-10^{3}$. As a consequence, the corrections can be directly obtained within the derivative expansion approach [31,34]. However, for the plasma prescription the derivative expansion clearly underestimates the correction, particularly for the largest radius shown in Fig. 2(b), and the leading-order correction is not proportional to $1 / R$.

In order to better understand the dependence on the sphere radius, we plot in Figs. 3(a) and 3(c) the force and force gradient corrections, respectively, as function of $1 / R$. For the plasma prescription, the force corrections are typically close to or above the percent level for the conditions of the experiment [23] where $1 / R=0.0242 \mu \mathrm{m}^{-1}$. More importantly for this experiment, the corrections to the force gradient are typically below $1 \%$ for submicrometer distances. In Figs. 3(b) and 3(d) the corrections to the force and force gradient, respectively, are scaled by $R / L$. While for the Drude prescription the data follow rather closely a $1 / R$ dependence, the results for the plasma prescription indicate a more singular approach to the PFA limit as $1 / R \rightarrow 0$.

In conclusion, we have shown that the Drude prescription for the Matsubara zero-frequency contribution leads to a weaker violation of the upper bound for the PFA correction derived experimentally by measuring the force gradient for different radii [20] than the dissipationless plasma prescription. This could have been expected, since dissipation is present in the gold coatings used in the experiments. However, all experiments performed with coated microspheres with aspect ratios $R / L \sim 10^{2}-10^{3}$ agree with the plasma prescription but not with the Drude prescription when the force variation with distance is analyzed for a given radius [21-25]. The proximity force approximation combined with the Drude prescription underestimates the experimental data for nonmagnetic materials, so that the correction calculated here brings the Drude prediction even further away from the experimental results. When taking the plasma prescription, the magnitude of the correction is significantly larger than predicted experimentally but still too small to degrade the quality of the comparison between the experimental data and the theory based on the plasma prescription. The theoretical results presented here, taking the sphere curvature fully into account, indicate that experiments probing the Casimir interaction beyond the PFA regime could provide new insight into the role of dissipation in Casimir physics.

We thank A. Canaguier-Durand, R. Guérout, A. Lambrecht, S. Reynaud, G. Bimonte, and F. D. Mazzitelli for discussions, and D. Dalvit for providing numerical data for the permittivity of gold along the 
imaginary frequency axis. We acknowledge support from CAPES and DAAD through the PROBRAL collaboration program. P. A. M. N. also thanks $\mathrm{CNPq}$ and FAPERJ for partial financial support.

[1] M. Bordag, G. L. Klimchitskaya, U. Mohideen, and V. M. Mostepanenko, Advances in the Casimir Effect (Oxford University Press, New York, 2009).

[2] G. L. Klimchitskaya, U. Mohideen, and V. M. Mostepanenko, The Casimir force between real materials: Experiment and theory, Rev. Mod. Phys. 81, 1827 (2009).

[3] R. Decca, V. Aksyuk, and D. López, Casimir force in micro and nano electro mechanical systems, Lect. Notes Phys. 834, 287 (2011).

[4] S. K. Lamoreaux, Progress in experimental measurements of the surface-surface Casimir force: Electrostatic calibrations and limitations to accuracy, Lect. Notes Phys. 834, 219 (2011).

[5] H. B. G. Casimir, On the attraction between two perfectly conducting plates, Proc. K. Ned. Akad. Wet. 51, 793 (1948).

[6] A. Lambrecht, P. A. Maia Neto, and S. Reynaud, The Casimir effect within scattering theory, New J. Phys. 8, 243 (2006).

[7] T. Emig, N. Graham, R. L. Jaffe, and M. Kardar, Casimir Forces between Arbitrary Compact Objects, Phys. Rev. Lett. 99, 170403 (2007).

[8] S. J. Rahi, T. Emig, N. Graham, R. L. Jaffe, and M. Kardar, Scattering theory approach to electrodynamic Casimir forces, Phys. Rev. D 80, 085021 (2009).

[9] P. A. Maia Neto, A. Lambrecht, and S. Reynaud, Casimir energy between a plane and a sphere in electromagnetic vacuum, Phys. Rev. A 78, 012115 (2008).

[10] T. Emig, Fluctuation-induced quantum interactions between compact objects and a plane mirror, J. Stat. Mech. (2008) P04007.

[11] A. Canaguier-Durand, P. A. Maia Neto, I. Cavero-Pelaez, A. Lambrecht, and S. Reynaud, Casimir Interaction between Plane and Spherical Metallic Surfaces, Phys. Rev. Lett. 102, 230404 (2009).

[12] A. Canaguier-Durand, P. A. Maia Neto, A. Lambrecht, and S. Reynaud, Thermal Casimir Effect in the Plane-Sphere Geometry, Phys. Rev. Lett. 104, 040403 (2010).

[13] A. Canaguier-Durand, P. A. Maia Neto, A. Lambrecht, and S. Reynaud, Thermal Casimir effect for Drude metals in the plane-sphere geometry, Phys. Rev. A 82, 012511 (2010).

[14] A. Canaguier-Durand, G.-L. Ingold, M.-T. Jaekel, A. Lambrecht, P. A. Maia Neto, and S. Reynaud, Classical Casimir interaction in the plane-sphere geometry, Phys. Rev. A 85, 052501 (2012).

[15] G. Bimonte and T. Emig, Exact Results for Classical Casimir Interactions: Dirichlet and Drude Model in the Sphere-Sphere and Sphere-Plane Geometry, Phys. Rev. Lett. 109, 160403 (2012).

[16] G. Bimonte, Classical Casimir interaction of a perfectly conducting sphere and plate, Phys. Rev. D 95, 065004 (2017).

[17] J. N. Israelachvili, Intermolecular and Surface Forces (Academic Press, London, 1991).
[18] S. K. Lamoreaux, Demonstration of the Casimir Force in the 0.6 to $6 \mu \mathrm{m}$ Range, Phys. Rev. Lett. 78, 5 (1997).

[19] A. O. Sushkov, W. J. Kim, D. A. R. Dalvit, and S. K. Lamoreaux, Observation of the thermal Casimir force, Nat. Phys. 7, 230 (2011).

[20] D. E. Krause, R. S. Decca, D. López, and E. Fischbach, Experimental Investigation of the Casimir Force beyond the Proximity-Force Approximation, Phys. Rev. Lett. 98, 050403 (2007).

[21] R. S. Decca, D. López, E. Fischbach, G. L. Klimchitskaya, D. E. Krause, and V. M. Mostepanenko, Tests of new physics from precise measurements of the Casimir pressure between two gold-coated plates, Phys. Rev. D 75, 077101 (2007).

[22] R. S. Decca, D. López, E. Fischbach, G. L. Klimchitskaya, D. E. Krause, and V. M. Mostepanenko, Novel constraints on light elementary particles and extra-dimensional physics from the Casimir effect, Eur. Phys. J. C 51, 963 (2007).

[23] C.-C. Chang, A. A. Banishev, R. Castillo-Garza, G. L. Klimchitskaya, V. M. Mostepanenko, and U. Mohideen, Gradient of the Casimir force between $\mathrm{Au}$ surfaces of a sphere and a plate measured using an atomic force microscope in a frequency-shift technique, Phys. Rev. B 85, 165443 (2012).

[24] A. A. Banishev, G. L. Klimchitskaya, V. M. Mostepanenko, and U. Mohideen, Demonstration of the Casimir Force between Ferromagnetic Surfaces of a Ni-Coated Sphere and a Ni-Coated Plate, Phys. Rev. Lett. 110, 137401 (2013).

[25] G. Bimonte, D. López, and R. S. Decca, Isoelectronic determination of the thermal Casimir force, Phys. Rev. B 93, 184434 (2016).

[26] A. Canaguier-Durand, Ph.D. thesis, Université Pierre et Marie Curie, 2011.

[27] D. S. Ether jr. et al., Probing the Casimir force with optical tweezers, Europhys. Lett. 112, 44001 (2015).

[28] L. P. Teo, M. Bordag, and V. Nikolaev, Corrections beyond the proximity force approximation, Phys. Rev. D 84, 125037 (2011).

[29] C. D. Fosco, F. C. Lombardo, and F. D. Mazzitelli, Proximity force approximation for the Casimir energy as a derivative expansion, Phys. Rev. D 84, 105031 (2011).

[30] G. Bimonte, T. Emig, R. L. Jaffe, and M. Kardar, Casimir forces beyond the proximity approximation, Europhys. Lett. 97, 50001 (2012).

[31] G. Bimonte, T. Emig, and M. Kardar, Material dependence of Casimir forces: Gradient expansion beyond proximity, Appl. Phys. Lett. 100, 074110 (2012).

[32] C. D. Fosco, F. C. Lombardo, and F. D. Mazzitelli, Derivative-expansion approach to the interaction between close surfaces, Phys. Rev. A 89, 062120 (2014).

[33] C. D. Fosco, F. C. Lombardo, and F. D. Mazzitelli, Derivative expansion for the electromagnetic Casimir free energy at high temperatures, Phys. Rev. D 92, 125007 (2015).

[34] G. Bimonte, Going beyond PFA: A precise formula for the sphere-plate Casimir force, Europhys. Lett. 118, 20002 (2017).

[35] F. C. Bohren and D. R. Huffman, Absorption and Scattering of Light by Small Particles (Wiley, New York, 1983), Chap. 4.

[36] S. Ambikasaran, A fast direct solver for dense linear systems, https://github.com/sivaramambikasaran/HODLR (2013). 
[37] S. Ambikasaran and E. Darve, An $\mathcal{O}(N \log N)$ fast direct solver for partial hierarchically semi-separable matrices, $\mathrm{J}$. Sci. Comput. 57, 477 (2013).

[38] I. Bogaert, B. Michiels, and J. Fostier, $\mathcal{O}(1)$ computation of Legendre polynomials and Gauss-Legendre nodes and weights for parallel computing, SIAM J. Sci. Comput. 34, C83 (2012).

[39] M. Hartmann and G.-L. Ingold (unpublished).

[40] Handbook of Optical Constants of Solids, edited by E. Palik (Academic Press, New York, 1998), Vol. I.

[41] A. Lambrecht and S. Reynaud, Casimir force between metallic mirrors, Eur. Phys. J. D 8, 309 (2000).

[42] S. Umrath, M. Hartmann, G.-L. Ingold, and P. A. Maia Neto, Disentangling geometric and dissipative origins of negative Casimir entropies, Phys. Rev. E 92, 042125 (2015).
[43] M. Boström and B. E. Sernelius, Thermal Effects on the Casimir Force in the $0.15 \mu \mathrm{m}$ Range, Phys. Rev. Lett. 84, 4757 (2000).

[44] R. Guérout, A. Lambrecht, K. A. Milton, and S. Reynaud, Derivation of the Lifshitz-Matsubara sum formula for the Casimir pressure between metallic plane mirrors, Phys. Rev. E 90, 042125 (2014).

[45] D. Garcia-Sanchez, K. Y. Fong, H. Bhaskaran, S. Lamoreaux, and H.X. Tang, Casimir Force and In Situ Surface Potential Measurements on Nanomembranes, Phys. Rev. Lett. 109, 027202 (2012).

[46] M. Bordag, G. L. Klimchitskaya, and V. M. Mostepanenko, Comment on Casimir Force and In Situ Surface Potential Measurements on Nanomembranes, Phys. Rev. Lett. 109, 199701 (2012). 